\title{
Complementarity and redundancy in the functional niche of cider apple pollinators
}

\author{
$\operatorname{Marcos}_{\text {MiñarRo }}{ }^{1}$, Daniel GARCíA ${ }^{2}$ \\ ${ }^{1}$ Servicio Regional de Investigación y Desarrollo Agroalimentario (SERIDA), Apdo. 13, E-33300, Villaviciosa, Asturias, \\ Spain \\ ${ }^{2}$ Departamento de Biología de Organismos y Sistemas, Universidad de Oviedo, y Unidad Mixta de Investigación en \\ Biodiversidad (CSIC-Uo-PA), C/Catedrático Rodrigo Uría s/n, E-33006, Oviedo, Asturias, Spain
}

Received 18 April 2018 - Revised 17 July 2018 - Accepted 3 September 2018

\begin{abstract}
The magnitude and the stability of pollination services in entomophilous crops both depend not only on honeybee management but also on the diversity of wild pollinators, which enables additive contributions and replacement of species. This work evaluated the functional niche of cider apple pollinators in Spain and found a highly heterogeneous community of pollinators in taxonomic and functional terms. Through different niche dimensions, our results suggest complementarity (i.e. niche differentiation) in the contribution of the various groups to pollination, through differences in abundance, foraging behaviour, diet and daytime and canopy distribution patterns, as well as redundancy (i.e. niche similarity) in their responses to environmental conditions. Thus, quantitative and qualitative complementarity would promote an additive effect of different insects on pollination, while similarity in environmental response would provide insurance against potential honeybee loss.
\end{abstract}

crop pollination / environmental response / foraging behaviour / Malus domestica / spatio-temporal patterns

\section{INTRODUCTION}

The importance of insect pollinators for global food production is now unquestionable (Klein et al. 2007; Garibaldi et al. 2013), and it will intensify under the scenario of increasing food needs and anthropogenic pollinator loss (Potts et al. 2010; Goulson et al. 2015). Farmers worldwide typically rely on the domesticated honeybee (Apis mellifera) to ensure pollination services in many crops (Aizen and Harder 2009; Garibaldi et al. 2013). However, depending exclusively on honeybee has been demonstrated to be ineffective,

Electronic supplementary material The online version of this article (https://doi.org/10.1007/s13592-018-0600-4) contains supplementary material, which is available to authorized users.

Corresponding author: M. Miñarro,

mminarro@serida.org

Handling editor: David Tarpy and even hazardous, as honeybees may be unable to cover the massive, short-term flowering of some crops (Brittain et al. 2013b; Grass et al. 2018), or could suffer severe declines due to colony collapse disorder or disease (vanEngelsdorp et al. 2009). In this context, wild pollinators would seem essential in overcoming honeybee constraints and to provide pollination insurance in the case of honeybee loss (Garibaldi et al. 2013; Rader et al. 2016). In fact, the magnitude and stability of pollination services in entomophilous crops is known to depend on the diversity and the species turnover of insect pollinators (Garibaldi et al. 2013; Rader et al. 2016; Winfree et al. 2018).

Ascertaining the role of pollinator diversity in crop pollination requires an understanding of the additive effects of various pollinators, as well as their potential for replacement following possible extinction. This can be achieved through studying the functional niche of pollinators (Blüthgen and Klein 2011), which represents the heterogeneity 
between insects contributing to pollination, by means of different dimensions. In this sense, functional niche may be addressed through insect abundance (Winfree et al. 2015), diet composition (Thomson and Goodell 2001), traits related to the function (Garibaldi et al. 2015) and foraging behaviours affecting access to flowers and pollen (Brittain et al. 2013a; Park et al. 2016). Also, functional niche may represent the response of a species to the filtering effects of environmental conditions, such as temperature, humidity or wind (e.g. Bartomeus et al. 2013; Brittain et al. 2013a). Thus, depending on the extent of segregation or overlap of their niche dimensions, pollinators may be considered as either complementary or redundant (Blüthgen and Klein 2011). This classification is not trivial given that complementarity in contributions may lead to additive effects across pollinators (Winfree 2013). In addition, complementarity in environmental responses may increase pollination resilience to climate change (Rader et al. 2013) while redundancy would ensure pollination stability across existing environmental gradients in the case of extinctions (Winfree and Kremen 2009; Winfree 2013). Although complementarity and redundancy are apparently two mutually exclusive scenarios, they can emerge simultaneously across different dimensions of the functional niche of pollinators.

Apple is a major fruit crop throughout the world (FAO 2018) and is heavily dependent on insect pollination (Klein et al. 2007). Pollinators determine apple production quantitatively, with 92 to $100 \%$ of apple yield relying on pollinators (Garratt et al. 2014; Miñarro and García 2016), as well as qualitatively, by increasing apple weight or sugar content (Geslin et al. 2017; Sapir et al. 2017). Besides honeybee, wild insects, including bumblebees, solitary bees and hoverflies, visit apple blossoms (e.g. Földesi et al. 2016; Martins et al. 2015; Russo et al. 2017). Importantly, the abundance and diversity of wild pollinators positively affect apple yield (Földesi et al. 2016; Martins et al. 2015; Blitzer et al. 2016). These positive effects seem to be related to the additive contributions of various pollinators that differ in abundance and quality depending on their foraging patterns (Thomson and Goodell 2001; Martins et al. 2015; Park et al. 2016). Although the functional variability of apple pollinators has been highlighted (Martins et al. 2015; Park et al. 2016; Russo et al. 2017), to date no study has focused on whole sets of apple pollinators by addressing simultaneously foraging differences and responses to environmental conditions (but see Vicens and Bosch 2000a, b; for a comparison A. melliferaOsmia cornuta ).

We therefore evaluate here the functional niche of apple pollinators, interpreting niche differences in terms of complementarity or redundancy. We studied the cider apple crops of the Asturias region in northern Spain, considering it to be a suitable model for evaluating pollinator functionality because: (1) a species-rich pollinator community is expected due to biogeographical and management factors (Miñarro et al. 2011); (2) the management of honeybee hives is not standardised in the locality, which allows for high variability in the density of managed pollinators and (3) regional weather conditions vary widely during apple bloom, including rain and low temperatures, providing large environmental gradients for pollinators. We hypothesised that honeybee and wild pollinators would show complementarity in both their contribution to pollination (Martins et al. 2015) and their response to climatic gradients (Bartomeus et al. 2013). Specifically, we sought to apply a multi-dimensional niche approach based on: (1) the structure and composition of the pollinator community; (2) the foraging behaviour and pollen-vs-nectar preferences of the different insect groups; (3) daytime temporal patterns and (4) responses to daytime temperature and humidity.

\section{MATERIALS AND METHODS}

\subsection{Study site}

Cider is a valuable traditional product with Protected Denomination of Origin status in Asturias (northern Spain). Indeed, almost the entire regional apple yield (up to 50,000 tons per year) is devoted to cider production. Apple orchards are planted with selected local cultivars that are tolerant to common apple diseases. In addition, as the cultural tolerance of growers to pests is high in general because aesthetic damage is of no 
importance for cider apples, the use of fungicides and insecticides is not common. These orchards are embedded in a highly variegated landscape and are typically surrounded by natural woody vegetation (Miñarro and Prida 2013; García et al. 2018). The low degree of agricultural intensification in these orchards and the surrounding landscape thus allow for high animal diversity within orchards, including natural enemies (Miñarro et al. 2011; García et al. 2018).

\subsection{Structure and composition of the pollinator community}

We aimed to identify the insect groups and species that comprised the pollinator community in the cider orchards, as well as to assess their abundances. To do so, we surveyed apple pollinators in 2015-2016 in 26 cider apple orchards in Asturias (see García et al. 2018, for a comprehensive description of sites). All orchards comprised several cultivars, but sampling was carried out on a single local cultivar, 'Regona', given that flower visits by pollinators may depend on cultivar (Garratt et al. 2016). Only two orchards managed pollination by introducing honeybee hives.

Both in 2015 and in 2016, each orchard was surveyed three times, each time by a different observer and at a different time in the day (from 1100 to $1300 \mathrm{~h}$, from 1300 to $1500 \mathrm{~h}$ or between 1500 and $1700 \mathrm{~h}$ ) in order to cover the maximum range of pollinator activity. Surveys were made under standard climatic conditions: dry vegetation, clear to lightly overcast skies, temperatures $>13{ }^{\circ} \mathrm{C}$, wind speed $<2.5 \mathrm{~m} \cdot \mathrm{s}^{-1}$ and when the 'Regona' cultivar was in full bloom. In each orchard, prior to full bloom we marked five target trees (at least $15 \mathrm{~m}$ from the edge to avoid a potential edge effect; Campbell et al. 2017), and then in each survey we observed a 1$\mathrm{m}$ diameter area of the canopy of each target tree for a period of $5 \mathrm{~min}$, recording the number of insect visits and the number of flowers in the selected area. In total, therefore, each orchard accounted for $75 \mathrm{~min}$ of observation (3 visits $\times 5$ trees $\times 5 \mathrm{~min}$ ) per year. To avoid disrupting floral visits, no insects were collected during the surveys and, therefore, we were only able to reliably identify the most easily recognised species (e.g. Apis mellifera, Bombus species, Andrena pilipes, Episyrphus balteatus, Oxythyrea funesta, etc.). Most visitors were, thus, assigned to one of the following groups: bumblebees, wild bees (categorised according to body size as either large, medium or small, when, respectively, bigger, similar or smaller than honeybees), hoverflies (predatory hoverflies with aphidophagous larvae, Eristalis hoverflies), flies (Diptera other than hoverflies), beetles, butterflies and 'other'. In order to better assess species richness, we also made separate assessments of apple pollinators by walking slowly along tree rows and catching all floral visitors, during a 10-min period during each survey event (i.e. a sum of 30 min per orchard per year). Captured specimens were identified in the laboratory.

\subsection{Foraging behaviour}

To estimate the differences in foraging behaviour between pollinator groups, we quantified visitation rate (the number of flowers a pollinator visits in a minute), how the insect approaches the flower (from the top or from the side), and tree canopy distribution (part of tree canopy, upper or lower, visited by insects). We also assessed inter-tree and inter-row movements (how often a pollinator left a tree to visit another, and whether the new tree was in a different row). Apple cultivars are auto-incompatible and thus, the frequent movement of pollinators between trees and tree rows is important to secure pollen transfer between cultivars (Ramírez and Davenport 2013).

During the observations of floral visits across the 26 orchards studied (see Section 2.1), we recorded the way in which the visitor approached flowers, distinguishing two behaviours that can affect the pollination outcome (Thomson and Goodell 2001, Martins et al. 2015; Russo et al. 2017): (1) 'top-working', when insects approached the top of the flower, increasing the probability of contacting both anthers and stigma and subsequently pollen removal; and (2) 'sideworking', when insects landed on the petals and approached the flower from the side, inserting their tongue in the base of stamens to collect nectar without coming into contact with anthers or stigma. Insects gathering pollen always contact the sexual organs of the flower (at least the 
anthers), and such visits can be considered legitimate in terms of pollination, as they contribute to pollen transfer. On the contrary, nectar-gathering insects can approach either from the side (illegitimate visit: nectar is collected without contribution to pollination) or from the top (legitimate visit). We calculated the frequency of topworking for each pollinator group.

Visitation rate and pollinator movements between trees and rows were sampled in 2016 only, in a single orchard with an abundant and rich pollinator community. To do this, any flower visitor detected during a slow walk along tree rows was visually tracked until lost, recording tracking time, number of flowers visited, and whether each visited flower was on the same or another tree and in the same or another tree row. Thus, we calculated the visitation rate (number of flowers visited per minute), the frequency of inter-tree movements (number of movements between trees per minute) and the frequency of inter-row movements (number of movements between rows per minute) for honeybees $(N=45)$, bumblebees (74), wild bees (75) and hoverflies (44). Flies and beetles were very passive and their movements were not recorded.

The distribution between the different parts of the tree canopy of pollinator visits was studied in five orchards in 2017. We stood in front of a given focal tree ( $N=72$; tree height from $\sim 3$ to $5 \mathrm{~m}$ ) for $1 \mathrm{~min}$, recording for each flower visit whether the insect visited the upper or the lower half of the canopy. In this way we were able to estimate the frequency of visits to upper and lower canopies for honeybees, bumblebees, wild bees, hoverflies, flies and beetles.

Differences between pollinator groups in terms of visitation rate and inter-tree and interrow movement rates were measured by means of Kruskal-Wallis tests (with post-hoc betweenpairs Mann-Whitney tests). We used Chisquare tests to analyse whether a given pollinator group differed from a random distribution in the frequency of top- versus side-working frequency, and in their frequency of visits to upper versus lower canopy. We also used Chi-square tests to compare the frequencies of top-working and of visits to upper canopy between pairs of pollinator groups.

\subsection{Trophic behaviour: pollen vs. nectar gathering}

Apple flower visitors may, as mentioned above, consume either one single resource (pollen or nectar) or two (both pollen and nectar). During the surveys of all 26 orchards (see Section 2.2), we also recorded the frequency of whether visitors were gathering pollen or nectar, or both when visitors gathered pollen and nectar from the same flower or from the consecutive flowers they visited. In such cases, we summed one to the frequency of each behaviour (pollen and nectar gathering). Departures from random in the frequency of pollen versus nectar gathering for pollinator groups, as well as differences between pairs of pollinator groups, were examined with Chi-square tests.

\subsection{Daytime temporal patterns}

In an experimental 1-ha orchard planted with a collection of more than 350 apple cultivars, we studied the distribution of flower visits at different times of the day by assigning visits to one of ten different hours (from 0900 to $1800 \mathrm{~h}$; hours hereafter). Observations were made in censuses on 22 days ( 9 days in 2014 and 13 in 2016). In each of the 220 censuses, one observer stood for $1 \mathrm{~min}$ in front of 20 different trees, counting and visually identifying all floral visitors belonging to six pollinator groups (honeybees, bumblebees, wild bees, hoverflies, beetles and flies). For each pollinator group and hour, we pooled all observations across days. We then made comparisons between pairs of pollinator groups of the distribution of observations for the different hours using KolmogorovSmirnov tests.

\subsection{Response to temperature and relative humidity}

We studied pollinator response to temperature and relative humidity within the same sampling set-up as in Section 2.5. The richness of cultivars in the experimental orchard enabled sampling to take place over the long overall blooming period resulting from the sequential bloom of different 
cultivars, which ensured that a variety of weather conditions prevailed on the observation days. The temperature and humidity data used in the analyses were from a weather station located $150 \mathrm{~m}$ away from the study site.

We used a spatially-explicit approach to evaluate the distribution of pollinator abundances in an environmental, bi-dimensional space of temperature and relative humidity. We estimated the degree of clumpiness (i.e. significant non-random aggregation of abundance) within this environmental space for each pollinator group, and quantified overlap/segregation between pollinator groups (i.e. concordance/discordance in the distribution across environmental space). We used spatial analysis by distance indices (SADIE, using Sadie Shell 2.0; Perry et al. 2002), to calculate, for each pollinator group, an aggregation index $\left(I_{a}\right)$ that measured clumpiness in the distribution of abundance (with values $I_{a}=1$ representing random, $I_{a}>1$ clumped, and $I_{a}<1$ regular distribution patterns). The degree of significance of $I_{a}$ (i.e. departure from a random distribution) was checked with a randomisation procedure (Perry et al. 2002). SADIE also provided a sampling point-level clustering index $(v)$, which quantified the degree to which the count at a given sampling point was contributing to the overall clumpiness. The clustering indexes of different pollinator groups may be correlated among themselves, in order to estimate overlap/segregation between pairs of groups, through an association index $X p$ (Perry and Dixon 2002) that ranges from +1 (complete spatial association) to -1 (complete dissociation, 0 indicating spatial independence), and whose statistical significance was quantified by the Dutilleul method (it corrects the amount of degrees of freedom in the presence of spatial autocorrelation).

In order to establish the environmental space for the analysis, we first considered the complete gradients of temperature and relative humidity recorded during the sampling. Temperature gradient (range: $11.8-22.4{ }^{\circ} \mathrm{C}$ ) was divided into 11 one-degree intervals $\left(11.5-12.4{ }^{\circ} \mathrm{C}, \ldots, 21.5-\right.$ $22.4{ }^{\circ} \mathrm{C}$; hereafter $12^{\circ}, \ldots, 22^{\circ}$ ) while we considered the relative humidity gradient $(34.8-91.8 \%)$ to comprise nine $5 \%$ intervals $(45.0-49.9 \%, \ldots$, 85.0-90\%; hereafter $45 \%, \ldots, 85 \%)$. Extreme values were incorporated into the lowest or highest intervals as appropriate. A total of 99 $(11 \times 9)$ combinations of temperature and relative humidity were possible, and data were available for 58 of them (due to the negative correlation between temperature and relative humidity; $r=-$ $0.566, P<0.0001, N=220$ ). After this, we considered a narrower scale with the central range of environmental conditions by using seven (15$\left.21^{\circ}\right)$ temperature and five $(50-70 \%)$ relative humidity intervals (data available for 29 of 35 possible combinations). Aggregation indexes were estimated for all groups of insects at the two study scales, as well as association indexes with respect to all possible pairings of pollinator groups.

\section{RESULTS}

\subsection{Pollinator community}

We recorded 3405 insect visits to apple flowers (Figure 1) in 2015 and 2016 across 26 orchards. Hymenopterans $(71.3 \%$ ) were the dominant order, followed by Diptera (26.7\%), Coleoptera (1.3\%) and Lepidoptera $(0.4 \%)$. Overall, the honeybee (Apis mellifera) was the most abundant taxon, followed by hoverfly, where Eristalis spp. dominated over predatory species. Among the wild bees, those categorised as small (mostly Halictidae) dominated over those categorised as medium or large (mostly Andrenidae), while Bombus terrestris was the most abundant bumblebee.

Of the 82 species of floral visitors identified (Table S1), Hymenoptera was the richest order (44 species), followed by Diptera (29 species). Wild bees, the richest group, were dominated by Halictidae and Andrenidae. The group of hoverflies, with 21 species, was the richest of the dipterans.

Apis mellifera was ubiquitous (Table S1) and also the most abundant ( $>50 \%$ of all pollinator visits in 21 of the 26 orchards; Figure S1). Another seven species (Bombus terrestris, Eristalis tenax, Episyrphus balteatus, Eristalis similis, Sphaerophoria scripta, Bombus pratorum and Andrena nigroaenea) occurred in ten or more sites, in both years. 


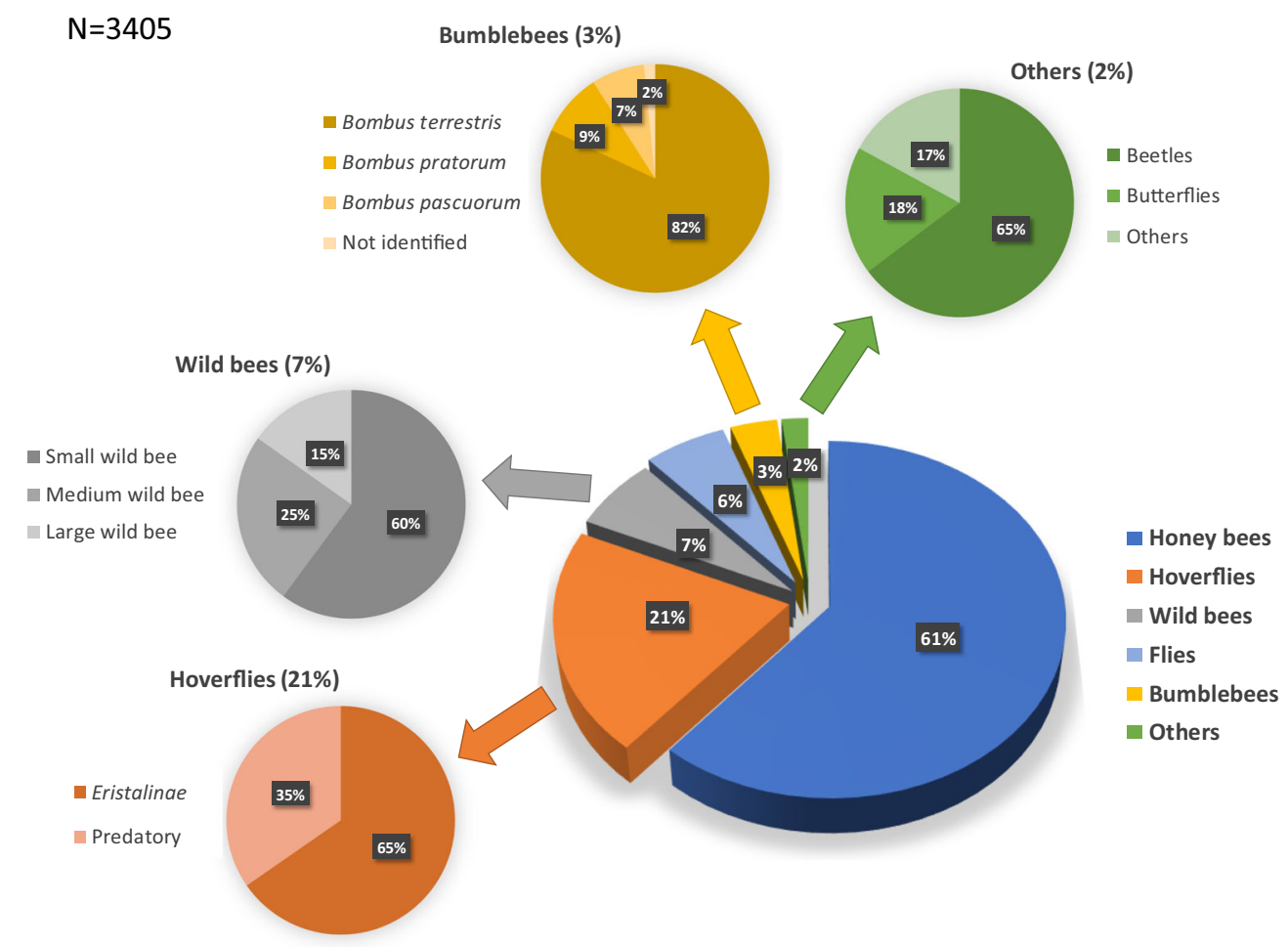

Figure 1. Community of insects visiting apple flowers in 26 cider orchards in Asturias (NW Spain) in 2015 and 2016.

\subsection{Foraging behaviour}

Pollinator groups differed in the number of apple flowers visited per minute (Kruskal-Wallis $\chi^{2}=144.7 ; \mathrm{df}=3 ; P<0.001 ;$ Mann-Whitney U $<429.0 ; P<0.001$ for all paired comparisons, but for wild bees-hoverflies, $U=1430.0 ; P=0.226$; Figure 2A). Bumblebees showed the highest visitation rate $\left(16.7 \pm 6.9\right.$ flowers $\cdot \mathrm{min}^{-1} ;$ mean \pm SD), almost double that of honeybees $(8.7 \pm$ 2.4 flowers $\cdot \min ^{-1}$ ). Wild bees and hoverflies were the visitors which spent most time per flower during their visits (they visited only $4.8 \pm 2.9$ and $4.7 \pm 4.4$ flowers $\cdot \min ^{-1}$, respectively).

Honeybees, bumblebees, hoverflies and wild bees mostly approached the flowers from the top ( $\chi^{2}$ tests; $P<0.001$ in all cases; Figure $2 \mathrm{~B}$ ), although with different frequencies (bumblebees = hoverflies $>$ wild bees $>$ honeybees; $\chi^{2}$ tests; $P<0.001$ in all paired combinations, but in bumblebee-hoverfly, $P=0.385$ ). Flies were detected resting in the petals or collecting nectar from the flower from the side in most cases $(P<0.001)$. No differences in the frequency of top- and sidevisits were detected for beetles $(P=0.095)$.

Pollinators differed in the frequency of changing tree $\left(\mathrm{KW} \chi^{2}=10.25\right.$; df $=3 ; P<0.017$; excluding flies and beetles due to lack of observations), with honeybees changing between trees more frequently than wild bees $(U=1300.5$; $P<0.017$; Table S2). In contrast, pollinators did not differ in the frequency of movement between tree rows $\left(\mathrm{KW} \chi^{2}=5.09 ; \mathrm{df}=3 ; P<0.160\right.$; Table S2).

Bumblebees, wild bees and hoverflies showed a preference in terms of tree canopy part (Figure 2C), with bumblebees being more frequent in the upper canopy $\left(\chi^{2}\right.$ test; $P=$ $0.008)$, with wild bees $(P=0.037)$ and hoverflies $(P=0.009)$ mostly appearing in the lower canopy. The bumblebee preference for the upper canopy was significantly stronger 

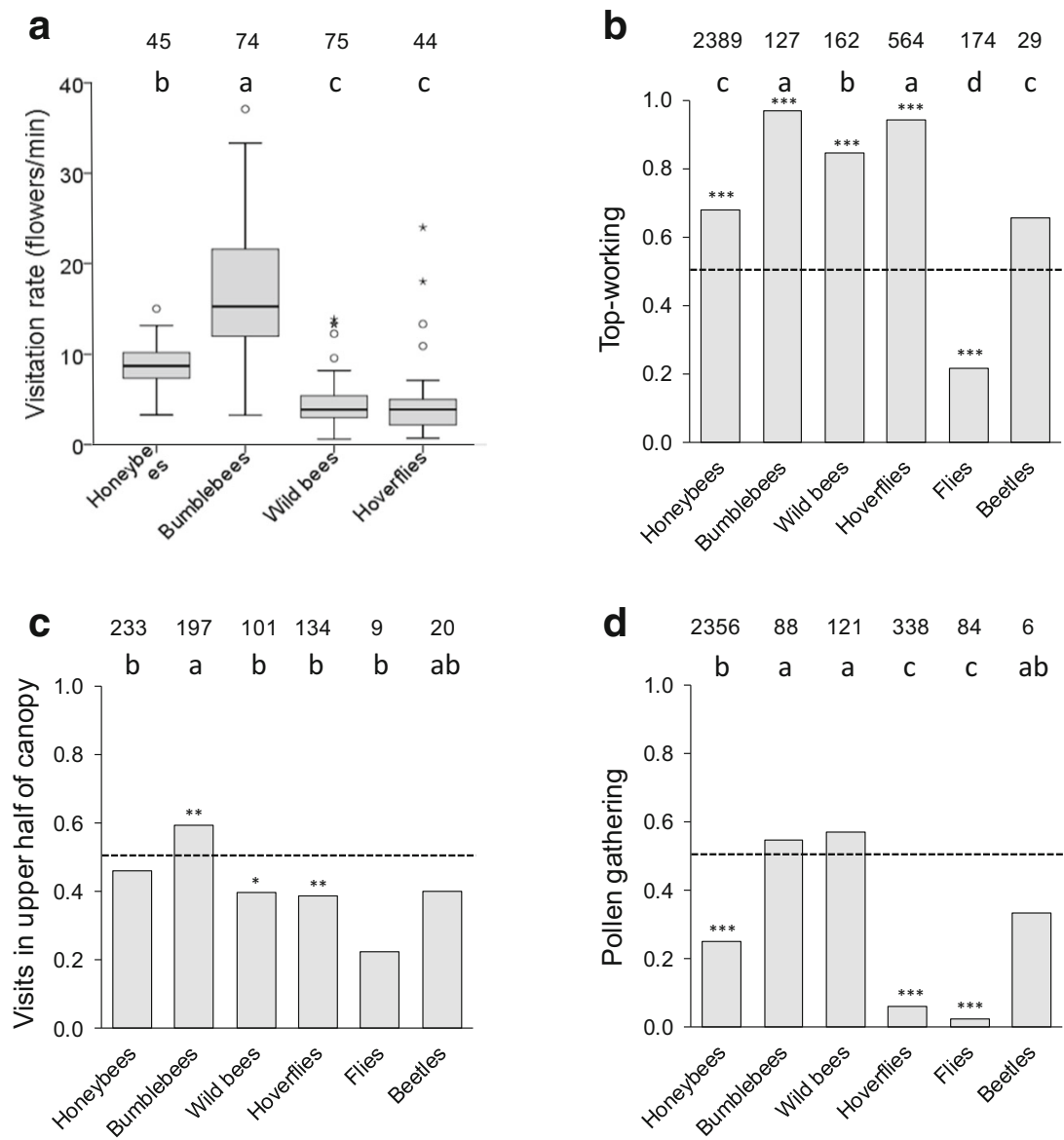

Figure 2. Foraging and trophic behaviour of cider-apple pollinators. A Visitation rate (number of flowers visited per minute), B frequency of top-working, $\mathbf{C}$ frequency of visits recorded in the upper half of the canopy and $\mathbf{D}$ frequency of individuals gathering pollen. Numbers at the top of each column indicate sample size. Different letters indicate differences between pollinator groups. Asterisks over the columns on $\mathbf{B}, \mathbf{C}$ and $\mathbf{D}$ reflect deviations from 1:1 for each pollinator (***: $P \leq 0.001, * *: P \leq 0.01, *: P \leq 0.05$ ). For $(\mathbf{A})$, boxplots indicate $25-75 \%$ quartiles (box boundaries), median (thick horizontal bar), largest and smallest observed values (whiskers), outliers (small circles) and extreme values (asterisks).

than in all other groups except beetles $\left(\chi^{2}\right.$ test $P=0.095$ for bumblebees-beetles).

\subsection{Trophic behaviour: pollen vs. nectar gathering}

There was a preference for collecting nectar in honeybees $\left(\chi^{2}\right.$ test, $\left.P<0.001\right)$, hoverflies $(P<0.001)$ and flies $(P<0.001)$ (Figure 2D). However, no statistically significant preferences for pollen or nectar were found for either bumblebees ( $\chi^{2}$ test, $\left.P=0.394\right)$ or wild bees $(P=0.122)$, although these groups did show a higher frequency of pollen gathering than the other pollinators ( $\chi^{2}$ tests; $P<0.001$ for all pair combinations). Similar differences emerged when comparing honeybees with hoverflies and flies $\left(\chi^{2}\right.$ tests; $P<0.001$ for all pair combinations).

\subsection{Daytime temporal patterns}

Sampling in the experimental orchard provided 10,725 visits to flowers. The pollinator community was dominated by honeybees $(67.9 \%)$, bumblebees $(15.8 \%)$, hoverflies 
(10.0\%), wild bees $(4.2 \%)$, beetles $(1.9 \%)$ and flies $(0.3 \%)$. Pollinator groups differed in how their daytime visits to flowers were distributed (Figure 3; Table S3). Namely, honeybees, hoverflies and flies were more active than other groups in the first hours (0900-1100 h), wild bees and beetles concentrated most of their activity in the central hours (1200 to $1500 \mathrm{~h}$ ) and bumblebees were the most vespertine group, with $40 \%$ of their visits occurring between 1600 and $1800 \mathrm{~h}$.

\subsection{Response to temperature and relative humidity}

\section{Aggregation indexes indicated significant} clumpiness in the abundances of pollinators across the sampled environmental space (Table I). Numbers of observations for all groups gradually increased from low temperature-high humidity to high temperature-low humidity conditions (Figure 4). An almost monotonic pattern was specially marked for honeybees. There was significant concordance (i.e. overlap) in the large-scale environmental distribution of all paired combinations of pollinator groups (Table II). Nevertheless, aggregation and concordance trends changed when the environmental space was narrowed. Although most groups still showed clumpy distributions at this smaller scale, fly and beetle abundances
Table I.. Index of aggregation $\left(I_{a}\right)$ measuring the degree of global clumpiness (i.e. aggregation of counts in space) and its significance $(P)$, indicating the degree in which the aggregated distribution departs from a random distribution. Data for large and small scales are shown

\begin{tabular}{llllll}
\hline & \multicolumn{2}{l}{ Large scale } & & \multicolumn{2}{l}{ Small scale } \\
\cline { 2 - 3 } Group & $I a$ & $P$ & & $I a$ & $P$ \\
\hline Honeybees & 3.83 & 0.001 & & 1.58 & 0.048 \\
Bumblebees & 3.77 & 0.001 & & 1.61 & 0.042 \\
Wild bees & 3.70 & 0.001 & & 1.96 & 0.006 \\
Hoverflies & 3.27 & 0.001 & & 1.78 & 0.018 \\
Flies & 2.44 & 0.001 & & 1.30 & 0.134 \\
Beetles & 2.45 & 0.013 & & 1.35 & 0.112 \\
\hline
\end{tabular}

appeared randomly distributed (Table I; Figure 4). More importantly, spatial concordance was not generalised, with bumblebee, fly and beetle abundance patches occurring independent of those of honeybees, as was also the case for wild bees and hoverflies (Table II).

\section{DISCUSSION}

In this study we characterised the whole community of insects visiting cider apple flowers across a regional extent in northern Spain using

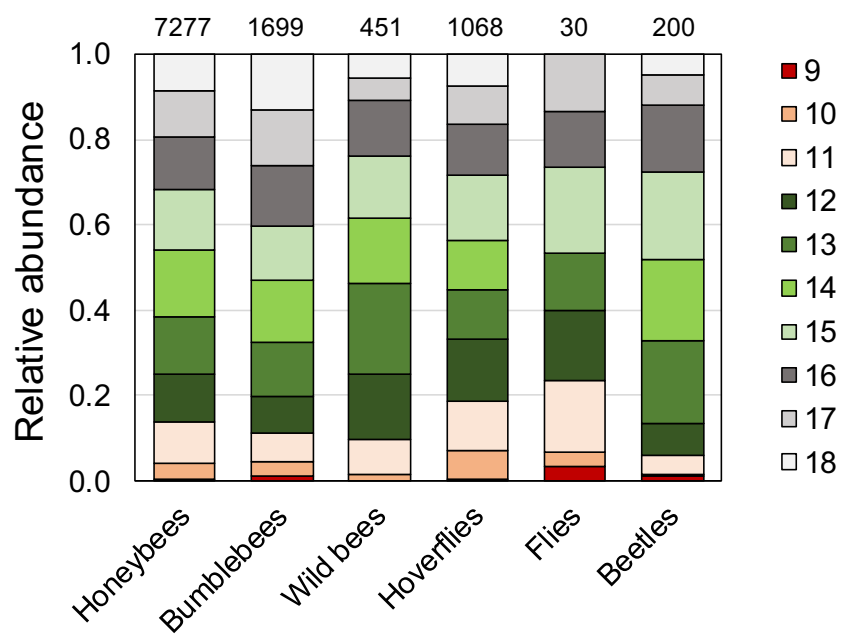

Figure 3. Distributions of frequencies of visits to apple flowers of each pollinator group throughout the day (from 0900 to $1800 \mathrm{~h}$ ). Numbers at the top of each column indicate number of pollinators observed in each group. 

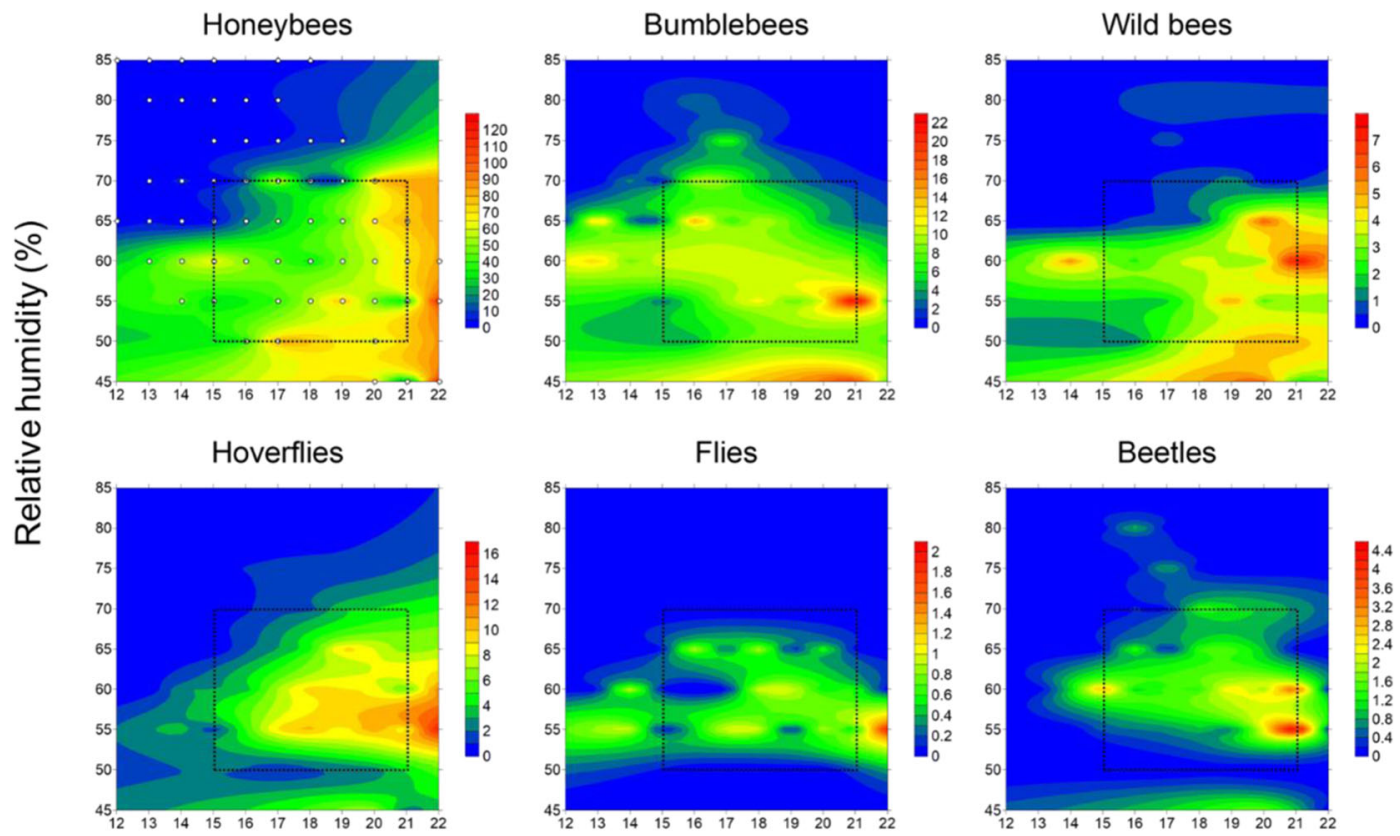

Temperature $\left({ }^{\circ} \mathrm{C}\right)$

Figure 4. Response surfaces of all the insects visiting apple flowers in relation to temperature and relative humidity. Dots in the first plot indicate the combinations of temperature and humidity registered during sampling (censuses). Coloured contours are interpolated from the number of visits in censuses. The colour scales represent the number of visits; note scale differences between insect groups. The inner rectangle indicates the small-scale observational space that considers a narrower, central range of environmental conditions $\left(15-21^{\circ} \mathrm{C}\right.$ temperature and $50-70 \%$ relative humidity). This small-scale subset accumulated more than $65 \%$ of sampled abundances for all insect groups.

non-manipulative sampling to evaluate insect behaviour and environmental responses which are important to pollination. We found a highly heterogeneous community of apple pollinators in both taxonomic and functional terms. Through various dimensions of the functional niche of pollination, our results suggest complementarity (i.e. niche segregation) in the different pollinator groups' contribution to pollination (through differences in abundance, foraging behaviour, diet and daytime distribution patterns), as well as scale-dependent redundancy (i.e. niche overlap) in their response to environmental conditions.

\subsection{The cider apple pollinator community}

Our study was based on direct observation and capture of flower visitors, hence providing conservative but unbiased information (compared with trap-sampling, Gibbs et al. 2017), and widened the scope beyond the usual focus of bees (see also Rader et al. 2016). We detected an abundant and rich community of flower visitors of over 80 species, of which $48 \%$ were non-bee pollinators. This is not a surprising result, as apple is an attractive flower resource due to the massive bloom (Grab et al. 2017) and easy accessibility to nectar and pollen (Ramírez and Davenport 2013). As such, pollinator pools very rich in wild species have also been found in apple crops in other regions (e.g. Martins et al. 2015; Földesi et al. 2016; Campbell et al. 2017).

In terms of visitation rate, the community studied was dominated by Apis mellifera, even though managed hives were present in only two of the orchards (see also Joshi et al. 2016). The common presence of non-professional, honeydevoted hives in farms around apple orchards, as 
Table II.. Index of association $\left(X_{p}\right)$ representing the concordance between clusters of two different count variables. It can be used as a measure of niche overlap (positively significant) or segregation (null or negatively significant). Values corresponding to the large scale are in the upper half-matrix, and those to the small scale in the lower halfmatrix (***: $P \leq 0.001, * *: P \leq 0.01, *: P \leq 0.05$, n.s.: $P>0.05$ )

\begin{tabular}{lllllll}
\hline & Honeybees & Bumblebees & Wild bees & Hoverflies & Flies & Beetles \\
\hline Honeybees & - & $0.692^{* * *}$ & $0.795^{* * *}$ & $0.795^{* * *}$ & $0.653^{* * *}$ & $0.587^{* * *}$ \\
Bumblebees & 0.087 n.s. & - & $0.745^{* * *}$ & $0.605^{* * *}$ & $0.634^{* * *}$ & $0.646^{* * *}$ \\
Wild bees & $0.624^{* * *}$ & $0.397^{*}$ & - & $0.792^{* * *}$ & $0.663^{* * *}$ & $0.651^{* * *}$ \\
Hoverflies & $0.409^{*}$ & $0.391^{*}$ & 0.148 n.s. & - & $0.789^{* * *}$ & $0.751^{* * *}$ \\
Flies & 0.199 n.s. & $0.584^{* *}$ & $0.461^{*}$ & $0.574^{*}$ & - & $0.729^{* * *}$ \\
Beetles & 0.052 n.s. & $0.667^{* * *}$ & $0.545^{* *}$ & $0.466^{* *}$ & $0.523^{* *}$ & - \\
\hline
\end{tabular}

well as the occurrence of some feral populations, would explain honeybee dominance in Asturian apple. Nevertheless, this dominance was not widespread: in several orchards, honeybee represented less than $20 \%$ of floral visitors, and in more than a third of orchards, almost $40 \%$ of visits were by wild pollinators (Figure S1). In sum, despite being a - quantitatively - honeybee-dominated crop, cider apple in Asturias benefits from a rich coterie of wild pollinators which are able to ensure high visitation rates when honeybee is rare. As a result, orchards with a low proportion of honeybees show visitation rates similar to those numerically dominated by honeybees (Figure S1).

\subsection{Foraging and trophic behaviour}

We recorded notable differences among pollinator groups in terms of foraging and trophic behaviour. First, pollinators differed in their speed of visiting flowers, with bumblebees being faster than honeybees and, especially, wild bees or hoverflies (see also Martins et al. 2015; Park et al. 2016, but see Vicens and Bosch 2000a). Second, pollinators differed in the way they approached flowers, with bumblebees, wild bees and hoverflies being mostly top-workers, whereas honeybees and flies showed increased rates of side-working. Similar differences have been noted in other regions (Vicens and Bosch 2000a, Martins et al. 2015; Park et al. 2016). Third, pollen vs. nectar foraging also varied among groups, with bumblebees and wild bees, again, showing higher frequencies of pollen gathering behaviour than honeybees, which is probably associated with their higher dependence on pollen for reproduction and larval development (Vaudo et al. 2015). Dipterans were, however, almost exclusively nectar foragers. This is to be expected because despite female hoverflies can occasionally feed on pollen to mature their ovaries and reach sexual maturity (Haslett 1989), they, unlike bees, do not use pollen for feeding larvae (e.g. Owen and Gilbert 1989). Nonetheless, hoverflies probably received and delivered high pollen loads as they usually accessed nectar from the flower top; thus their hairy body would come into contact with the flowers' reproductive parts (e.g. Eristalis spp.). In honeybees, side-working and nectargathering behaviour have been related with lower pollen removal and deposition (Thomson and Goodell, 2001).

In this study, we found honeybees moving between trees with a higher frequency than other groups. The lower tolerance of honeybees to interspecific aggressive encounters in trees could underpin this pattern (Brittain et al. 2013b; Sapir et al. 2017). Nevertheless, we did not observe differences between pollinator groups in the frequency of movements between tree rows (but see Campbell et al. 2017). We did however register some spatial segregation of pollinators within tree canopies, with bumblebees being more frequently observed in the upper part, wild bees and hoverflies at the bottom, while honeybees were observed throughout the canopy. Irrespective of the mechanism which provokes segregation (e.g. interspecific competition; Brittain et al. 2013a), it 
may have a positive impact on apple crop yield as different parts of the blossom canopy would be setting fruit, thanks to different insects (Brittain et al. 2013a).

\subsection{Daytime temporal patterns and response to temperature and relative humidity}

Flowers received pollinators during the entire $10 \mathrm{~h}$ over which observations were conducted, although there were differences in how the different groups distributed their visits throughout the day: wild bees, hoverflies and flies were early visitors, whereas bumblebees showed a higher frequency of afternoon visits. This temporal segregation could have positive consequences for apple pollination, leading to better coverage of the daily interval of stigmatic receptivity and opening of anthers (Herrera 1990). Among the potential mechanisms underlying the differences found may be differences in life histories (e.g. some flies overnight on petals taking advantage of radiation reflection early in the morning; Vicens and Bosch 2000b), thermoregulation ability (small-sized wild bees may depend more on day central temperatures; Bishop and Armbruster 1999), and also interspecific competition (Brittain et al. 2013a).

Our spatial approach showed a significant overlap among pollinator groups in their response to temperature and relative humidity. All pollinator groups increased in abundance as conditions became warmer and dryer, and were able to cope with the recorded range of weather conditions (temperature: $11.8-22.4{ }^{\circ} \mathrm{C}$, relative humidity: $34.8-91.8 \%$ ). The absence of differences may result, nevertheless, from the limitation of the observational space to detect the relevance of extreme or rare values, given the strength of the large-scale, monotonic gradient based on the negative correlation between temperature and humidity. In fact, when the response to environmental conditions was analysed at a finer scale (i.e. reducing the magnitude of the environmental gradient), some differences between pollinator groups arose under hot and dry conditions, with bumblebees prevailing over honeybees, and flies and beetles showing distributions less affected by the environmental gradients. Although we cannot exclude competitive avoidance as an alternative explanation, such a fine-scale segregation in the environmental space may also be due to different thermoregulation constraints between species (Rader et al. 2013).

\subsection{Complementarity and redundancy in the cider apple pollination niche}

Our approach seeks to interpret the contribution of different flower visitors to cider apple pollination by integrating different dimensions of functional niche. Importantly, we found significant differences between pollinator groups in all niche components assumed to impact on the magnitude of pollination: abundance, visitation rate, trophic and foraging behaviour and spatio-temporal distribution. Thus, we consider that, although numerically dominated by Apis mellifera, cider apple in Asturias is far from being a honeybee-dependent crop. This is because wild pollinators show diverse behaviours that may qualitatively compensate for their usually lower abundances (see also Garratt et al. 2016). For example, the high visitation rate of the less common bumblebees would mean a high proportion of flowers pollinated by this group. Similarly, a higher frequency of legitimate visits (i.e. an enhanced effectiveness of pollination) is expected from the predominantly top-working behaviour of bumblebees, wild bees and hoverflies, as well as from the higher frequency of pollen collection behaviour by bumblebees and wild bees. Therefore, by means of quantitative and qualitative complementarity, we may expect an additive effect of the different insect groups on pollination (Martins et al. 2015; Garratt et al. 2016).

Unlike the behavioural dimensions, and in contrast to our initial hypothesis, no clear evidence of niche segregation in the pollinator response to environmental conditions was found in our study. Some differences in the environmental distribution were observed at the fine scale, for example between honeybees and bumblebees, flies and beetles. These subtle differences could also mean additive contributions to pollination, especially for non-bee pollinators (Rader et al. 2016). Neverthe 
less, the wide overlap among pollinator taxa suggested a redundant response along the larger environmental gradients studied here. This also means that, despite the frequent much higher abundance of honeybees, when wild pollinators are considered all together, they covered all the conditions in which honeybees occurred. Thus, in a scenario of honeybee locally extinct (e.g. by colony collapse disorder), the local community of wild insects could fulfil pollination requirements over the complete environmental gradients covered by the managed species. This sort of redundancy may be thought to be positive for the stability of apple yield, when providing ecological insurance under events of honeybee collapse and high meteorological variation during apple bloom (Vicens and Bosch 2000b). In fact, both types of events are particularly relevant in the context of climate change (Bartomeus et al. 2013; Rader et al. 2013).

As a final remark, we would highlight that, as has been shown for other crops (Garibaldi et al. 2013; Rader et al. 2016), both wild insects and honeybees are probably necessary to optimise the global service of pollination in cider apple. We encourage, therefore, actions to promote wild pollinator diversity through habitat management (floral cover in orchards and surrounding woody hedgerows; Miñarro and Prida 2013; Dicks et al. 2016; Campbell et al. 2017), in parallel with honeybee management regimes that are non-harmful for wild species (Geldmann and González-Varo 2018).

\section{ACKNOWLEDGEMENTS}

We thank R. Martínez-Sastre, A. Núñez, C. Guardado, D. Luna, K. Twizell and A. Somoano for technical support; R. Lendrum for linguistic advice; Campoastur S. Coop. Asturiana for orchard selection; the orchard owners for providing access to their properties; and L.O. Aguado, P. Alvarez and J. Ortiz for taxonomic support. Funding was provided by grants INIA-RTA2013-00139-C03-01 (Ministerio de Economía, Industria y Competitividad (MinECo) and Fondo Europeo de Desarrollo Regional) to $\mathrm{MM}$ and PCIN2014-145-C02-02 (MinECo; EcoFruit project BiodivERsA-FACCE2014-74) and CGL2015-68963C2-2-R (MinECo/FEDER) to DG.

\section{AUTHOR CONTRIBUTION}

MM and DG conceived and designed the research, collected field data, analysed the data and wrote the manuscript, which both authors have approved.

\section{COMPLIANCE WITH ETHICAL STANDARDS}

Conflict of interest The authors declare that they have no potential conflict of interest.

Complémentarité et redondance dans la niche fonctionnelle des pollinisateurs de pommes à cidre

Pollinisation des cultures / réponse environnementale / comportement alimentaire / Malus domestica / modèles spatio-temporels

Komplementarität und Redundanz in der funktionellen Nische von Mostapfelbestäuber

Kulturpflanzenbestäubung, Umweltantwort, Foragierverhalten, Malus domestica, räumlichzeitliche Muster

\section{REFERENCES}

Aizen, M. A., Harder, L. D. (2009) The global stock of domesticated honey bees is growing slower than agricultural demand for pollination. Curr. Biol. 19 (11), 915-918.

Bartomeus, I., Park, M. G., Gibbs, J., Danforth, B. N., Lakso, A. N., Winfree, R. (2013) Biodiversity ensures plant-pollinator phenological synchrony against climate change. Ecol. Lett. 16 (11), 1331-1338.

Bishop, J. A., Armbruster, W. S. (1999) Thermoregulatory abilities of Alaskan bees: effects of size, phylogeny and ecology. Funct. Ecol. 13 (5), 711-724.

Blitzer, E. J., Gibbs, J., Park, M. G., Danforth, B. N. (2016) Pollination services for apple are dependent on diverse wild bee communities. Agric. Ecosyst. Environ. 221, $1-7$.

Blüthgen, N., Klein, A.M. (2011) Functional complementarity and specialisation: the role of biodiversity in plant-pollinator interactions. Basic Appl. Ecol. 12 (4):282-291.

Brittain, C., Kremen, C., Klein, A. M. (2013a) Biodiversity buffers pollination from changes in environmental conditions. Glob. Change Biol. 19 (2), 540-547. 
Brittain, C., Williams, N., Kremen, C., Klein, A. M. (2013b) Synergistic effects of non-Apis bees and honey bees for pollination services. Proc. Roy. Soc. B 280 (1754), 20122767.

Campbell, A. J., Wilby, A., Sutton, P., Wäckers, F. L. (2017) Do sown flower strips boost wild pollinator abundance and pollination services in a springflowering crop? A case study from UK cider apple orchards. Agric. Ecosyst. Environ. 239, 20-29.

Dicks, L.V., Viana, B., Bommarco, R., Brosi, B., del Coro Arizmendi, M. et al. (2016) Ten policies for pollinators. Science 354 (6315), 975-976.

FAO (2018) Food and Agriculture Organization of the United Nations, http://www.fao.org/faostat/en/\#data/QC (Accessed 24 February 2018).

Földesi, R., Kovács-Hostyánszki, A., Kőrösi, Á., Somay, L., Elek, Z. et al. (2016) Relationships between wild bees, hoverflies and pollination success in apple orchards with different landscape contexts. Agric. Forest Entomol. 18 (1), 68-75.

García, D., Miñarro, M., Martínez-Sastre, R. (2018) Birds as suppliers of pest control in cider apple orchards: Avian biodiversity drivers and insectivory effect. Agric. Ecosyst. Environ. 254, 233-243.

Garibaldi, L.A., Steffan-Dewenter, I., Winfree, R., Aizen, M.A., Bommarco, R. et al. (2013) Wild pollinators enhance fruit set of crops regardless of honey bee abundance. Science 339 (6127), 1608-1611.

Garibaldi, L.A., Bartomeus, I., Bommarco, R., Klein, A.M., Cunningham, S.A. et al. (2015) Trait matching of flower visitors and crops predicts fruit set better than trait diversity. J. Appl. Ecol. 52 (6),1436-1444.

Garratt, M. P. D., Breeze, T. D., Jenner, N., Polce, C., Biesmeijer, J. C., Potts, S. G. (2014) Avoiding a bad apple: Insect pollination enhances fruit quality and economic value. Agric. Ecosyst. Environ. 184, 3440.

Garratt, M. P. D., Breeze, T. D., Boreux, V., Fountain, M. T., McKerchar, M. et al. (2016) Apple pollination: demand depends on variety and supply depends on pollinator identity. PloS one 11 (5), e0153889.

Geldmann, J., González-Varo, J.P. (2018) Conserving honey bees does not help wildlife. Science 359 (6374), 392-393.

Geslin, B., Aizen, M. A., Garcia, N., Pereira, A. J., Vaissière, B. E., Garibaldi, L. A. (2017) The impact of honey bee colony quality on crop yield and farmers' profit in apples and pears. Agric. Ecosyst. Environ. 248, 153-161

Gibbs, J., Joshi, N. K., Wilson, J. K., Rothwell, N. L., Powers, K. et al. (2017) Does passive sampling accurately reflect the bee (Apoidea: Anthophila) communities pollinating apple and sour cherry orchards?. Environ. Entomol. 46 (3), 579-588.

Goulson, D., Nicholls, E., Botías, C., Rotheray, E.L. (2015) Bee declines driven by combined stress from parasites, pesticides, and lack of flowers. Science 347 (6229), 1255957.

Grab, H., Blitzer, E. J., Danforth, B., Loeb, G., Poveda, K. (2017) Temporally dependent pollinator competition and facilitation with mass flowering crops affects yield in co-blooming crops. Sci. Rep. 7, 45296.

Grass, I., Meyer, S., Taylor, P. J., Foord, S. H., Hajek, P., Tscharntke, T. (2018) Pollination limitation despite managed honeybees in South African macadamia orchards. Agric. Ecosyst. Environ. 260, 11-18.

Haslett, J.R. (1989) Adult feeding by holometabolous insects: pollen and nectar as complementary nutrient sources for Rhingia campestris (Diptera: Syrphidae). Oecologia 81, 361-363.

Herrera, C. M. (1990) Daily patterns of pollinator activity, differential pollinating effectiveness, and floral resource availability, in a summer-flowering Mediterranean shrub. Oikos 58, 277-288.

Joshi, N. K., Otieno, M., Rajotte, E. G., Fleischer, S. J., Biddinger, D. J. (2016) Proximity to woodland and landscape structure drives pollinator visitation in apple orchard ecosystem. Front. Ecol. Evol. 4, 38.

Klein, A.M., Vaissiere, B.E., Cane, J.H., Steffan-Dewenter, I., Cunningham, S.A., Kremen, C., Tscharntke, T. (2007) Importance of pollinators in changing landscapes for world crops. Proc. Roy. Soc. B 274 (1608), 303-313.

Martins, K. T., Gonzalez, A., Lechowicz, M. J. (2015) Pollination services are mediated by bee functional diversity and landscape context. Agric. Ecosyst. Environ. 200, 12-20.

Miñarro, M., García, D. (2016) Manzana, kiwi y arándano: sin insectos no hay frutos ni beneficios. Tecnología Agroalimentaria 18, 4-8.

Miñarro, M., Prida, E. (2013) Hedgerows surrounding organic apple orchards in north-west Spain: potential to conserve beneficial insects. Agric. Forest Entomol. 15 , 382-390.

Miñarro, M., Dapena, E., Blázquez, M.D. (2011) Guía ilustrada de las enfermedades, las plagas y la fauna beneficiosa del cultivo del manzano. Ed. SERIDA. Asturias.

Owen, J., Gilbert, F. S. (1989) On the abundance of hoverflies (Syrphidae). Oikos 55, 183-193.

Park, M. G., Raguso, R. A., Losey, J. E., \& Danforth, B. N. (2016). Per-visit pollinator performance and regional importance of wild Bombus and Andrena (Melan ?drena) compared to the managed honey bee in New York apple orchards. Apidologie 47 (2), 145-160.

Perry, J. N., Dixon, P.M. (2002) A new method to measure spatial association for ecological count data. Ecoscience 9, 133-141.

Perry, J. N., Leibhold, A. M., Rosenberg, M. S., Miriti, M., Jakomulska, A., and Citron-Pousty, S. (2002) Illustrations and guidelines for selecting statistical methods for quantifying spatial pattern in ecological data. Ecography 25, 578-600.

Potts, S.G., Biesmeijer, J.C., Kremen, C., Neumann, P., Schweiger, O., Kunin, W.E. (2010) Global pollinator declines: trends, impacts and drivers. Trends Ecol. Evol. 25 (6), 345-353.

Rader, R., Reilly, J., Bartomeus, I., Winfree, R. (2013) Native bees buffer the negative impact of climate 
warming on honey bee pollination of watermelon crops. Glob. Change Biol. 19, 3103-3110.

Rader, R., Bartomeus, I., Garibaldi, L. A., Garratt, M. P., Howlett, B. G. et al. (2016) Non-bee insects are important contributors to global crop pollination. PNAS 113 (1), 146-151.

Ramírez, F., Davenport, T. L. (2013) Apple pollination: A review. Sci. Hort. 162, 188-203.

Russo, L., Park, M. G., Blitzer, E. J., Danforth, B. N. (2017) Flower handling behavior and abundance determine the relative contribution of pollinators to seed set in apple orchards. Agric. Ecosyst. Environ. 246, 102108.

Sapir, G., Baras, Z., Azmon, G., Goldway, M., Shafir, S. et al. (2017) Synergistic effects between bumblebees and honey bees in apple orchards increase cross pollination, seed number and fruit size. Sci. Hort. 219, $107-117$.

Thomson, J.D., Goodell, K. (2001) Pollen removal and deposition by honeybee and bumblebee visitors to apple and almond flowers. J. Appl. Ecol. 38, 10321044.

vanEngelsdorp, D., Evans, J. D., Saegerman, C., Mullin, C., Haubruge, E. et al. (2009) Colony collapse disorder: a descriptive study. PloS one 4 (8), e6481.

Vaudo, A.D., Tooker, J.F., Grozinger, C.M., Patch, H.M. (2015) Bee nutrition and floral resource restoration. Curr. Opin. Insect Sci. 10, 133-141.
Vicens, N., Bosch, J. (2000a) Pollinating efficacy of Osmia cornuta and Apis mellifera (Hymenoptera: Mega ?chilidae, Apidae) on 'Red Delicious' apple. Environ. Entomol. 29, 235-240.

Vicens, N., Bosch, J. (2000b) Weather-dependent pollinator activity in an apple orchard, with special reference to Osmia cornuta and Apis mellifera (Hymenoptera: Megachilidae and Apidae). Environ. Entomol. 29, 413-420.

Winfree, R. (2013) Global change, biodiversity, and ecosystem services: What can we learn from studies of pollination? Basic Appl. Ecol. 14 (6), 453-460.

Winfree, R., Kremen, C. (2009) Are ecosystem services stabilized by differences among species? A test using crop pollination. Proc. Roy. Soc. B 276 (1655), 229237.

Winfree, R., W Fox, J., Williams, N. M., Reilly, J. R., Cariveau, D. P. (2015) Abundance of common species, not species richness, drives delivery of a real-world ecosystem service. Ecol. Lett. 18 (7), 626-635.

Winfree, R., Reilly, J. R., Bartomeus, I., Cariveau, D. P., Williams, N. M., Gibbs, J. (2018). Species turnover promotes the importance of bee diversity for crop pollination at regional scales. Science 359(6377), 791-793. 